\title{
The outbreak of the SARS-CoV-2 Omicron variant make imperative the adoption of telerehabilitation in the Bulgarian health care system
}

\author{
Jannis Papathanasiou (1,2), Yana Kashilska (3), Hristo Bozov (4), Ivo Petrov (5), \\ Stefano Masiero (6)
}

(1) Department of Kinesitherapy, Faculty of Public Health "Prof. Dr. Tzecomir Vodenicharov, DSc”, Medical University of Sofia, Bulgaria; (2) Department of Medical Imaging, Allergology \& Physiotherapy, Faculty of Dental Medicine, Medical University of Plovdiv, Bulgaria; (3)Medical Center for Outpatient Rehabilitation and Sport Medicine, Plovdiv, Bulgaria; (4) Complex Oncologic Center of Burgas, Faculty of Public Health and Health Care, University prof. Assen Zlatarov, Burgas, Bulgaria; (5) Clinic of Cardiology and Angiology, Acibadem City Clinic Cardiovascular Center University Hospital, Sofia, Bulgaria; (6) Physical Medicine and Rehabilitation Unit, Department of Neurosciences, University of Padua, Padua, Italy.

This article is distributed under the terms of the Creative Commons Attribution Noncommercial License (CC BY-NC 4.0) which permits any noncommercial use, distribution, and reproduction in any medium, provided the original author(s) and source are credited.

\begin{abstract}
The rapid spread of the highly contagious Omicron variant of SARS-CoV-2 globally will challenge the accessibility and the delivery of physical and rehabilitation medicine (PRM) services. Many health care systems throughout the world performed effective reforms such as the transition to telerehabilitation (TR). In Bulgaria, TR is still not regulated by law, and terms such as teleconsultation and tele-education have not yet been introduced. The adoption of TR in the Bulgarian health care system will undoubtedly increase the accessibility to rehabilitation treatment for a larger group of Bulgarian patients with various neurological, cardiorespiratory, musculoskeletal, and oncological conditions and will significantly contribute to the PRM services modernization in Bulgaria.
\end{abstract}

Key Words: Bulgarian Society of Physical and Rehabilitation Medicine (BGSPRM); Omicron variant of SARS-CoV-2; Telerehabilitation (TR); TeleHealth Center.

Eur J Transl Myol 32 (1): 10355, 2022 doi: 10.4081/ejtm.2022.10355

The rapid spread of the highly contagious SARS-CoV2 Omicron variant, even among highly vaccinated populations, will challenge the accessibility and the delivery of physical and rehabilitation medicine (PRM) services in many countries throughout the world as well as in Bulgaria. The currently prevalent SARS-CoV-2 Omicron variant will continue to create collateral damages and negative consequences to both, people with disabilities and rehabilitation facilities ${ }^{1,2}$ Many health care systems throughout the world performed effective reforms such as the transition to telerehabilitation (TR) in response to the call for action of World Health Organization (WHO) and several PRM scientific organizations, for strengthening the affected rehabilitation services during the COVID-19 pandemic. $^{3}$ TR is a subfield of telemedicine (TM), defined as the provision of rehabilitation services to patients at a remote location via telecommunication technologies and teleconference apps such as Skype (Microsoft Co), Facebook Messenger (Facebook Inc), Viber (Rakuten Group Inc), and WhatsApp (Facebook Inc). ${ }^{4}$ TR can play an important role in public health emergencies, such as the current outbreak of SARS-CoV-2 Omicron variant, while promoting flexibility and allowing greater continuity of rehabilitation care for patients with spinal cord injuries, ${ }^{2}$ as well as patients with cardiorespiratory, musculoskeletal, and oncological conditions. ${ }^{5,6}$ Recent studies suggest that TR visits are safe and effective treatment approach for people with disabilities, ${ }^{2}$ and has several advantages over in-person rehabilitation such as early access to rehabilitation, reduced health care costs, improved treatment adherence, and health-related quality of life. ${ }^{7}$ TR can be provided in a variety of different ways, including two-way real-time visits with audio, video, or both, asynchronous e-visits, virtual check-ins, remote evaluations of recorded videos or images and telephone assessment and management services. ${ }^{8}$ Moreover, it can be combined with in-person rehabilitation or used alone as a comprehensive hybrid approach even in case either of COVID-19. ${ }^{9}$ A clinicaltrials.gov search shows over 340 clinical trials on TR, which confirm the increased scientific and 
research interest regarding TR globally. ${ }^{10}$ Interestingly, in a resource-limited country such as Bulgaria with almost 250 PRM physicians, ${ }^{11}$ and a total of 6.365 beds for inpatient PRM services $(9.0$ beds per 10.000 population (NCPHA, 2017), ${ }^{12}$ TR is still not regulated by law, and terms such as teleconsultation and teleeducation have not yet been introduced. ${ }^{13}$ Unlike the response of several PRM scientific societies and academies worldwide ${ }^{4,9}$ that recommended the TR as an effective alternative way on delivery of rehabilitation, no activities (e.g., webinars, online references) supporting the TR, were provided by the Bulgarian Society of Physical and Rehabilitation Medicine (BGSPRM) during the pandemic. ${ }^{11}$ Recently, an evidence-based position statement issued from PRM experts suggesting the integration of TR in the new developed clinical pathway No. 267, regarding the rehabilitation of post-COVID-19 syndrome was rejected by the Bulgarian Ministry of Health $(\mathrm{BMoH})^{14}$ and the National Health Insurance Fund (NHIF). ${ }^{15}$ Nonetheless, we identified several barriers such as the lack of legislative framework, data privacy, patient safety, reimbursement, and NHIF coverage, as well as the lack of established national TR guidelines, which limiting its implementation in the Bulgarian rehabilitation practice. We acknowledge that COVID-19 is not a short-term problem and the emergence of the SARS-CoV-2 Omicron variant, may accelerate the reorganization and modernization of rehabilitation services in Bulgaria, assessed as less effective and less digital. ${ }^{12,13}$ Therefore, Bulgarian Regulation amendments (BG) No. 9/2019, laying down the package of medical activities reimbursed by the NHIF are crucial and will enable the use of telecommunication tools for diagnosis, treatment, and rehabilitation in Bulgaria. We advise the BGSPRM to take the leadership role in the establishment of a National Telehealth Center which will be dedicated to the development and implementation of TM solutions adapted to all types of rehabilitation. Bulgarian health policymakers, and experts from BGSPRM must consider TR as a new approach to common rehabilitation practice, not only in specific situations like the COVID-19 emergency. In this context, a step forward by the BGSPRM should be the establishment of TR national guidelines which must be broadly supported also by other Bulgarian scientific and professional organizations such as the Bulgarian Orthopedic and Traumatology Association (BOTA), Bulgarian Society of Neurology (BSN), Bulgarian Society of Cardiology (BSC), Bulgarian Society of Lung Diseases (BSLD), and Bulgarian Cancer Scientific Society (BCSS). Faced with new waves of the COVID19 pandemic and the prospect of new restrictions, TR in rehabilitation practice is mandator in Bulgaria to increase accessibility to rehabilitation treatment for patients with various neurological, cardiorespiratory, musculoskeletal, and oncological conditions who are often disadvantaged by several barriers and will contribute significantly to the modernization of PRM services in Bulgaria.

\section{List of acronyms}

BCSS - Bulgarian Cancer Scientific Society

BGSPRM: Bulgarian Society of Physical and Rehabilitation Medicine

BMoH - Bulgarian Ministry of Health

BOTA - Bulgarian Orthopedic and Traumatology Association

BSC - Bulgarian Society of Cardiology

BSLD - Bulgarian Society of Lung Diseases

BSN - Bulgarian Society of Neurology

COVID-19: SARS-CoV-2 disease

NHIF: National Health Insurance Fund

PRM - physical and rehabilitation medicine

SARS-CoV-2: Severe acute respiratory syndrome coronavirus- 2

TR - telerehabilitation

WHO - World Health Organization

\section{Authors contributions}

JP, YK, HB, IP, SM were involved in the conception, drafting and critical revision of the manuscript. All authors approved the final edited typescript.

\section{Acknowledgments}

Thanks to all the peer reviewers for their opinions and suggestions.

\section{Funding}

This research received no external funding.

\section{Conflict of Interest}

The authors have no conflicts of interest to report.

\section{Ethical Publication Statement}

We confirm that we have read the Journal's position on issues involved in ethical publication and affirm that this report is consistent with those guidelines.

\section{Corresponding Author}

Prof. Dr. Jannis Papathanasiou

Department of Kinesitherapy, Faculty of Public Health "Prof. Dr. Tzecomir Vodenicharov, DSc", Medical University of Sofia, Bulgaria.

Department of Medical Imaging, Allergology \& Physiotherapy, Faculty of Dental Medicine Medical University of Plovdiv, Bulgaria.

ORCID iD: 0000-0003-2557-5508

E-mail: giannipap@yahoo.co.uk

E-mails and ORCID iD of co-authors

Yana Kashilska: iana kashilska@abv.bg ORCID iD: 0000-0002-8859-7934

Hristo Bozov: hrbozov@yahoo.com ORCID iD: 0000-0001-7897-2051

Ivo Petrov: petrovivo@hotmail.com

ORCID iD: 0000-0002-9989-0730 
Stefano Masiero: stef.masiero@unipd.it ORCID iD: 0000-0002-0361-4898

\section{References}

1. Negrini S, Grabljevec K, Boldrini P, Kiekens C, Moslavac S, Zampolini M, Christodoulou N. Up to 2.2 million people experiencing disability suffer collateral damage each day of Covid-19 lockdown in Europe. Eur J Phys Rehabil Med. 2020 Jun; 56(3):361-365. doi: 10.23736/S19739087.20.06361-3.

2. Alexander M. It's Corona Calling: Time for Telerehabilitation! J Frailty Sarcopenia Falls. 2020 Dec 1;5(4):86-88. doi: 10.22540/JFSF-05-086. eCollection 2020 Dec.

3. Lugo-Agudelo LH, Cruz Sarmiento KM, Spir Brunal MA, Velásquez Correa JC, Posada Borrero AM, Fernanda Mesa Franco L, Di Dio Castagna Ianini R, Ramírez Pérez Lis PA, Vélez CM, Patiño Lugo DF, Gutenbrunner C. Adaptations for rehabilitation services during the COVID-19 pandemic proposed by scientific organizations and rehabilitation professionals. J Rehabil Med. 2021 Sep 16; 53(9): jrm00228. doi: 10.2340/165019772865.

4. Kilova K, Kitova T, Kasnakova P. Telemedicine in help of rehabilitation in the conditions of COVID19. Health Policy Technol. 2021 Jun; 10(2):100508. doi: 10.1016/j.hlpt.2021.100508.

5. Shukla H, Nair SR, Thakker D. Role of telerehabilitation in patients following total knee arthroplasty: evidence from a systematic literature review and meta-analysis. $\mathrm{J}$ Telemed Telecare. 2017 Feb; 23(2):339-346. doi: 10.1177/1357633X16628996.

6. Batalik L, Filakova K, Batalikova K, Dosbaba F. Remotely monitored telerehabilitation for cardiac patients: A review of the current situation. World J Clin Cases. 2020 May 26; 8(10):1818-1831. doi: 10.12998/wjcc. v8.i10.1818.
7. Pastora-Bernal JM, Estebanez-Pérez MJ, MolinaTorres G, García-López FJ, Sobrino-Sánchez R, Martín-Valero R. Telerehabilitation Intervention in Patients with COVID-19 after Hospital Discharge to Improve Functional Capacity and Quality of Life. Study Protocol for a Multicenter Randomized Clinical Trial. Int J Environ Res Public Health. 2021 Mar 12; 18(6):2924. doi: 10.3390/ijerph18062924.

8. Prvu Bettger J, Resnik LJ. Telerehabilitation in the Age of COVID-19: An Opportunity for Learning Health System Research. Phys Ther. 2020 Oct 30;100(11):1913-1916.

9. D Leochico CF. Adoption of telerehabilitation in a developing country before and during the COVID19 pandemic. Ann Phys Rehabil Med. 2020 Nov; 63(6):563-564. doi: 10.1016/j.rehab.2020.06.001.

10. ClinicalTrials.gov. Available at: https://clinicaltrials.gov. (Accessed: January 21, 2022).

11. Bulgarian Association of Physical and Rehabilitation Medicine. Available at: https://bgsprm.com/. (Accessed: January 5, 2022).

12. Dimova A, Rohova M, Koeva S, Atanasova E, Koeva-Dimitrova L, Kostadinova T, Spranger A. Bulgaria: Health System Review. 2018 Sep; 20(4):1-230.

13. Dzhafer N, Papathanasiou J. Telerehabiltation and COVID-19. Time for reorganization of rehabilitation services in Bulgaria? Inspiro 2021; 60(2):9-12.

14. State Gazette of the Republic of Bulgaria. Available dv.parliament.bg/DVWeb/showMaterialDV. jsp?idMat=158089. (Accessed: January 5, 2022).

15. National Health Insurance Fund of Bulgaria. Available at: https://www.en.nhif.bg. (Accessed: January 5, 2022).

Submission: January 6, 2022 Revision submitted: January 21, 2022 Accepted for publication: January 22, 2022 